\title{
4. CONTRIBUTION À L’ÉTUDE DE LA VÉGÉTATION STEPPIQUE DU MAROC ORIENTAL: TRANSECT JERRADA - FIGUIG
}

\author{
Souâd BEN EL MOSTAFA, Benyounes HALOUI \& Abdelabasset BERRICHI
}

Contribution study to the steppique vegetation of Eastern Morocco: Transect Jerrada-Figuig.

Mots clés. Maroc oriental, steppe, écologie, Stipa tenacissima.

Key words. Eastern Morocco,Steppe, ecologia, Stipa tenacissima.

En botanique comme en faunistique, les recherches scientifiques concernent surtout les régions riches en espèces végétales et animales telles que les forêts, les prairies. Les zones arides, par contre, tiennent encore à des lacunes dans les connaissances scientifiques et techniques, tel est le cas de notre dition. En effet, cette région a été peu parcourue par les naturalistes et ce n'est que récemment qu'elle a fait l'objet de certaines recherches générales (Haloui, 1991; Kahouadji, 1995; Bouraada, 1996).

Dans ces zones arides, la charge pastorale associée à l'exploitation irrationnelle des ressources végétales par l'homme (nappes alfatières) constitue une importante cause de dégradation des formations végétales. De plus la composition floristique de ces formations, en particulier pour les espèces annuelles, subit d'importantes variations en fonction des précipitations annuelles. Cette inconstante floristique amène à des difficultés au niveau de la méthode d'échantillonnage. Par ailleurs la présence des espèces annuelles exprime le moment et l'intensité des pluies qui définissent respectivement le nombre d'individus et l'appartenance biogéographique des espèces et seules les plantes pérennes sont le reflet permanent de la station.

La région prospectée (fig. 1) correspond à:

- une partie des hauts plateaux (sud de Jerrada - Aîn Béni Mathar - Tendrara) qui constitue une vaste formation tabulaire située à une altitude moyenne de $1000 \mathrm{~m}$,

- la région de Bouârfa - Figuig, caractérisée par des chaînes montagneuses qui

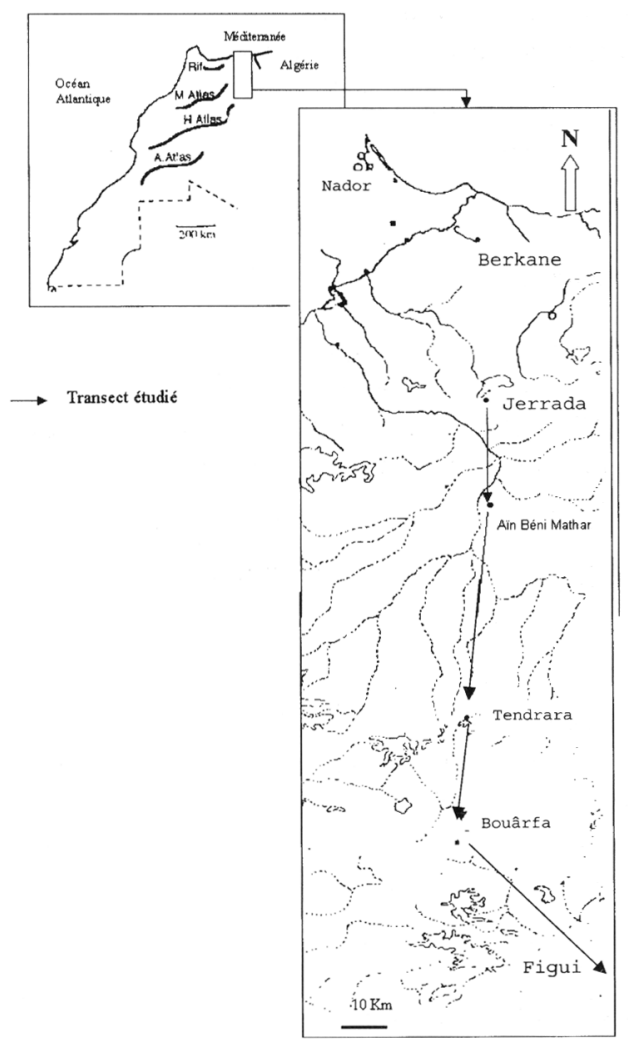

Figure 1. Localisation géographique de la région étudié (axe Jerrada-Figuig.). 
sont presque dépourvues de végétation. Elle correspond à la partie la plus orientale du grand Atlas et à l'extrême Est aux premières formations de l'Atlas Saharien. Ces massifs sont traversés par de vastes plaines.

Du point de vue climatique, le territoire étudié est caractérisé par un climat méditerranéen semi-aride, aride et saharien avec des précipitations comprises entre 364 $\mathrm{mm} / \mathrm{an}$ au nord et $100 \mathrm{~mm} / \mathrm{an}$ au sud. Du point de vue phytogéographique (Kaabeche, 1996), il appartient aux domaines suivants :

- le domaine maghrébin steppique qui correspond aux hautes plaines algéromarocaine, dont une partie de notre dition, et aux hautes plaines steppiques tunisiennes à climat continental. Il est caractérisé par une saison sèche relativement longue (6 à 7 mois) et des précipitations irrégulières de l'ordre de 200 à $400 \mathrm{~mm} / \mathrm{an}$.

- le domaine méditerranéen saharien qui constitue approximativement la limite septentrionale du Sahara avec des précipitations inférieures à $200 \mathrm{~mm}$. Celui ci s'inscrit dans la partie la plus méridionale de la région étudiée.

En vue d'analyser et de définir les groupements végétaux, nous avons utilisé pour notre échantillonnage la méthode classique «sigmatiste» de Braun Blanquet. Après une reconnaissance générale du territoire qui nous a permis de choisir les stations d'échantillonnage, nous avons pris en considération sur le terrain des variables d'ordre stationnel à savoir le type physionomique de la végétation exprimé par les espèces vivaces dominantes. Au sein d'un même paysage végétal apparemment homogène, nous avons tenu compte de l'hétérogénéité du milieu : colline, dépression...etc.

Pour chaque station d'échantillonnage, l'exécution des relevés a été choisie en fonction de certains critères. Elle a été réalisée de telle manière à s' approcher de la courbe de saturation aire-espèce. Nous avons choisi, comme l'a fait
(Djebaili, 1984), une aire minimale de l'ordre de $100 \mathrm{~m}^{2}$ pour l'ensemble des formations végétales indépendamment des variations de pluie et de milieux. Au sein d'une même formation végétale, le nombre de relevés floristiques est d'autant plus important que celle-ci occupe une grande surface et les conditions du milieu sont plus variées. Pour chaque relevé, les espèces sont affectées d'un coefficient d'abondance-dominance (Braun Blanquet, 1952). Nos relevés floristiques ont été effectués au cours des printemps et automnes des années 1998 et 1999. Ces années sont caractérisées malheureusement par une remarquable sécheresse qui a posé des difficultés au niveau de l'appréciation de la sociabilité des individus et par suite la définition des associations.

La détermination des espèces végétales a été effectuée à l'aide de la Nouvelle Flore de l'Algérie (Quèzel \& Santa, 1962-1963), la Flore de l'Afrique du Nord (Maire, 1952-1980), la Petite Flore des régions arides du Maroc occidental (Negre, 1961) et la Flore du Sahara (Ozenda, 1977). Des échantillons des espèces déterminées sont conservées dans l'herbier du département de Biologie de la Faculté des Sciences d'Oujda.

L'ensemble des données sont rassemblées dans un tableau à double entrées dont les colonnes correspondent aux relevés et les lignes représentent les espèces. Ce tableau est constitué par 52 relevés décrits surtout par des espèces perennes. Il a fait l'objet d'une analyse factorielle des correspondances (A.F.C.) qui constitue la méthode statistique la plus appropriée pour mettre en évidence des groupements végétaux. Le logiciel utilisé est MacMul (Thioulouse, 1989)

Les trois premiers axes de l'AFC englobent $36 \%$ de l'inertie totale avec des contributions partielles respectives de $14 \%$, $12 \%$ et $10 \%$. Ces valeurs relativement faibles ne compromettent en aucun cas le pouvoir discriminant des axes factoriels de ce type 
d'AFC (Foucart, 1985).

L'analyse du plan factoriel (F1 xF2) (fig.2) permet la mise en évidence de 7 groupements répartis suivant un gradient nord-sud représenté par l'axe F1:

1- Pseudosteppe ou forêt pré-steppique (Groupe A)

Sur les piémonts des versants sud des monts de Jerrada el sous un bioclimat essentiellement semi-aride. l'alfa couvre presque complètement le sol. Toutefois, une analyse floristique montre qu'il s'agit en fat d'une formation très dégradée de thuya. Il y a. en effel, présence de quelques pieds de Tetraclinis articulata Masters, Pistacia lentiscus L. Olea europaea L.. Juniperus oxycedrus L. Quèzel (1999) signale que, d'une manière générale, les forêts pré-steppiques sont actuellement répandues tant au maghreb qu'en méditerranée orientale en bioclimat semi-aride voire aride. Negre (1959) considère ce type de végétation comme un territoire de l'étage aride. II est le résultat de contraintes édaphoclimatiques ou d'une exacerbation des actions anthropiques. Dans notre cas, l'exposition de ces monts vers les influences sahariennes en pius d'une exploitation irrationnelle du bois seraient sans doute à l'origine de l'installation de ce type de végétation.

2- Steppe à Stipa tenacissima L. (Groupe B)

Ce rype de végétation connu sous le nom de steppe aride occupe au maghreb des superficies considérables avec 4500000 ha sur la méseta du Maroc oriental (Kaabeche, 1996). Dans le territoire étudié, l'alfa s'étend sur la plus grande partie et occupe les hautes plaines, les collines et les bas et mi-versants. Elle se dévellope en étages semi-aride et aride et occupe des sols variés. Selon le degré de dégradation, elle forme des nappes plus ou moins denses depuis le sud des monts de Jerrada jusqu'aux montagnes de Bouârfa là où les précipitations annuelles varient d'environ 250 $\mathrm{mm} / \mathrm{an}$ à $150 \mathrm{~mm} / \mathrm{an}$. Plus au sud, l'alfa ne

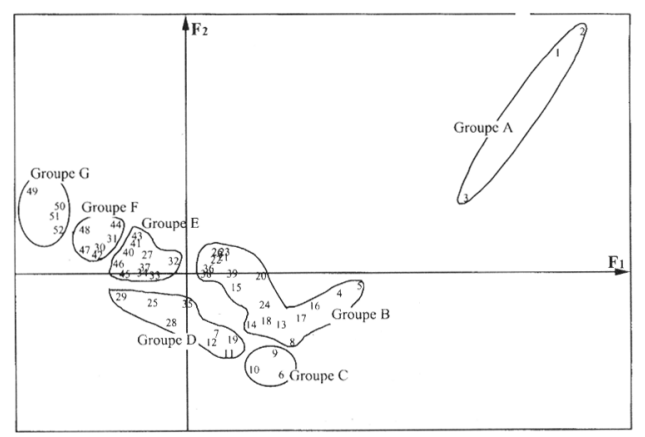

Figure 2. Projection des relevés floristiques selon le plan factoricl (F1x F2).

persiste qu'en pieds isolés au bord des lits d'oueds.

Aussi faut - il constater que cette essence se rencontre sous divers faciès. Elle peut former des peuplements purs ou être accompagnée par d’autres espèces principalement Artemisia herba-alba Asso., Lygeum spartum L., Noaea mucronata Asch. \& Schw., Peganum harmala L.. Atractylis humilis L. et A. serratuloides Sich. Vers sa limite inférieure qui coincide approximativement avec la barrière que constitue ies chaînons de Bouârfa, d'autres types despeces de souche saharienne s'infiltrent à la formation d'alfa, il s'agit principaiement de Thymeiaea microphylla Coss. \& Dur., Haloxylon scoparium Pomel, Anabasis aretioides Bunge, Zilla macroptera Coss.

En fonction de certaines conditions édaphiques, l'alfa peut être éliminée et remplacéc par l'armoise blanche ou la sparte déterminant ainsi des types de formation distincts. Dans le stade ultime de dégradation. elle est remplacée par $P$. harmala qui domine le paysage.

3- Steppe à Artemisia herba-alba Asso. (Groupe C)

Sur les hauts plateaux, cette formation occupe des surfaces moins importantes que 
l'alfa et remplace celle-ci dans dépressions non salées et les glacis à sol généralement limono-argileux, peu perméable où l'eau est facilement retenue. Elle se dévellope également sur sol caillouto-terreux ou sablo-limoneux. Sur le plan floristique, l'armoise blanche constitue un groupement avec: Lygeum spartum, N. mucronata, A. serratuloides, Stipa tenacissima L., P. harmala et Salvia verbenaca L. Sur le plan phytosociologique, ce groupement appartient à l'association des Noaeo-Lygeetum (Djebaili, 1984).

4- Steppe à Lygeum spartum L. (Groupe D)

Cette formation est rencontrée principalement en étage aride sur les glacis et les dépressions non salées et trouve son maximum d'expansion sur les dépôts sablonneux où elle forme des microdunes. Parmi les principales espèces qui constituent un groupement avec la sparte on cite $A$. herbaalba, N. mucronata, S. tenacissima et $A$. serratuloides. Ce groupement appartient à l'association des Noaeo-Lygeetum (Djebaili, 1984). Plus au sud, la sparte s'associe avec d'autres types de plantes formant ainsi un groupement plus xérique que le premier; il s'agit principalement de Helianthemum lippii Pers., A. serratuloides, Haloxylon scoparium Pomel, Z. macroptera, Anvillea radiata Coss. et Dur.

Djebaili (1984) a signalé qu'en Algérie et au Maroc, la sparte semble préférer les sols à texture sablo-limoneuse (Sable $>60 \%$ et Limon<10\%) sur croûte calcaire. En Tunisie, par contre, Le Houerou (1966) et Boukhris (1973) la classent parmi les gypsophytes.

5- Steppe à Haloxylon scoparium Pomel (Groupe E)

Cette formation occupe des surfaces assez vastes dans la région de Bouârfa. Elle colonise surtout les plateaux horizontaux et les dépressions à sol limono-argileux ou limonosableux. Sur le plan climatique, la steppe à $H$. scoparium se trouve aussi bien dans l'étage aride que saharien et constitue la limite sud de l'aire d'extension de l'alfa comme c'est le cas au niveau du bassin de Guercif et Outat El haj (Haloui, 1991). Dans cette aire, l'afa s'installe sur les pentes (monts de Bouârfa) alors que $H$. scoparium se contonne sur les replats. Sur le plan floristique, vers sa limite nord, $H$. scoparium se trouve associé à $N$. mucronata, L. spartum, S. tenacissima, A. serratuloide; plus au sud, d'autres types d'espèces à affinité saharienne apparaissent telles que : $A$. aretioides, Marrubium deserti (De Noé) Coss., Anvillea radiata Coss. \& Dur. et Thymelaea microphylla Coss. \& Dur.

6- Steppe à Anabasis aretioides Bugen (Groupe F)

Cette formation caractérise la végétation des sols rocheux et couvre des surfaces assez vastes entre Bouârfa et Figuig sous un bioclimat saharien. Elle est appelée aussi pseudosteppe diffuse à A. aretioides. (Guinet, 1954). Parmi les principales espèces rencontrées, il y a $Z$. macroptera, A. serratuloides, H. scoparium, M. deserti, H. lippii, Limoniastrum feei Batt. et Th. microphylla.

7- Steppe à Aristida pungens Desf. (Groupe G)

La steppe à A ristida pungens Desf. comme celle d' A. aretiodes représente une végétation typiquement saharienne. Elle constitue une végétation caractéristique des dunes sableuses dans la région de Figuig qu'on appelle aussi ergs. Elle est formée principalement par A. pungen, Launaea arborescens Maire, Th. microphylla, Retama retam Webb., Z . macroptera, Randonia africana Coss. et Genista saharae Coss. \& Dur.

L'analyse floristique de la végétation steppique du territoire étudié permet de reconnaître trois grands ensembles végétaux qui se succédent dans l'espace suivant un gradient de continentalité orienté Nord-Sud. Le premier correspond à des vestiges de forêts ou pseudosteppe qui se développent dans la partie la plus septentrionale de la zone étudiée. Il est constitué d'alfa et de restes d'arbres ou 
d'arbustes tels que le thuya, l'oxycèdre et le lentisque. Le second rassemble la végétation steppique des hauts plateaux caractérisée principalement par l'alfa. Suivant son degré de dégradation ou les conditions du milieu, l'alfa peut être remplacée, localement ou sur des étendues assez vastes, par d'autres types d'espèces qui constituent alors des groupements physionomiquement distincts; il s'agit de $L$. spartum et de $A$. herba-alba. Le troisième ensemble végétal rassemble une végétation plus xérique rappellant les zones sahariennes. On distingue une végétation des sols rocheux ou regs à base d'A. aretioides et une végétation caractéristique des dunes sableuses ou ergs constituée principalement d'A. pungens. La steppe à $H$. scoparium assure le passage de la steppe à $S$. tenacissima prospérant en bioclimat aride aux steppes diffuses se dévellopant en bioclimat saharien.

\section{ANNEXE}

Les stations de présence de chaque espèce sont représentées entre parenthèses : (1) Jerrada ; (2) Ain Béni mathar ; (3) Tendrara ; (4) Bouârfa ; (5) Figuig

\section{ANCARDIACÉES}

Pistacia atlantica Desf. (1, 2, 3, 4, 5)

Pistacia lentiscus L. (1)

\section{APOCYNACÉES}

Nerium oleander L.(5)

\section{BORAGINACÉES}

Echium plantagineum L. (2)

Echium pycnanthum subsp humile Jah. \& Maire (5)

\section{CARYOPHYLLACÉES}

Dianthus crinitus Sm. (3)

Gymnocarpos decander Forsk.(5)

Paronychia chlorothyrsa Murb.(3)

Silene villosa Forsk. (5)

\section{CHÉNOPODIACÉES}

Agathophora alopecuroides Bunge (4)
Anabasis aretioides Bunge $(4,5)$

Anabasis articulata subsp. oropediorum Maire $(4,5)$

Haloxylon scoparium Pomel $(2,3,4,5)$

Noaea mucronata Asch. \& Sch. $(2,3,4)$

Salsola tetragona Del. $(4,5)$

Salsola vermiculata $\mathrm{L}$. $(3,4)$

\section{CISTACÉES}

Helianthemum croceum Pers. (1)

Helianthemum lippii Pers. (2, 3, 4, 5)

Helianthemum pilosum Pers. $(1,2)$

Helianthemum ruficomum Spr. $(3,4,5)$

\section{COMPOSÉES}

Anvillea radiata Coss. \& Dur. $(4,5)$

Artemisia heba alba Asso.(1, 2, 3, 4)

Atractylis humilis L. $(1,2,3,4)$

Atractylis serratuloides Sieb. $(2,3,4,5)$

Echinops spinosus L. $(4,5)$

Launaea arborescens Maire $(4,5)$

Micropus bombycinus Lag. $(1,2)$

Nolletia chrysocomoides Coss. (5)

\section{CONVOLVULACÉES}

Convolvulus althaeoides L. $(1,4)$

Convolvulus supinus Coss. \& Kral. (5)

\section{CRUCIFERES}

Alyssum linifolium Willd.(3)

Alyssum granatense Boiss. \& Reut. (1)

Alyssum scutigerum Dur.(3)

Carrichtera annua D.C. (1)

Eruca vesicaria Cav. $(1,2,3)$

Farsetia hamiltonii Royle (5)

Farsetia ramosissima Hochst.(5)

Malcolmia aegyptica Spr.(5)

Matthiola parviflora $\mathrm{R}$. Br. (1)

Moricandia arvensis D.C (4)

Notoceras bicorne Amo. $(1,2)$

Zilla macroptera Coss. $(4,5)$

CUPRESSACÉES

Juniperus oxycedrus L. (1)

Tetraclinis articulata Masters. (1)

CAPPARIDACÉES

Capparis spinosa L.(5)

\section{DIPSACACÉES}

Scabiosa stellata L. (1, 2, 4) 


\section{EUPHORBIACÉES}

Euphorbia calyptrata Coss. \& Dur $(3,5)$

Euphorbia falcata L.(1)

Euphorbia guyoniana Boiss. \& Reut.(5)

FAGACÉES

Quercus rotundifolia Lam. (1)

\section{GÉRANIACÉES}

Erodium guttatum Will. (2)

Erodium malacoides L'Hérl. $(1,3)$

\section{GRAMINÉES}

Ammochloa subacaulis Coss. \& Dur. (2)

Aristida plumosa L. (5)

Aristida pungens Desf. (5)

Bromus madritensis L. (2)

Bromus rubens L. $(1,2,3)$

Cutandia dichotoma Trab. (1)

Cynodon dactylon Pers.(1)

Dactylis glomerata L. (1)

Lygeum spartum L. (2, 3, 4, 5)

Phragmites communis Trin.(5)

Schismus barbatus Thell. $(1,2,4)$

Stipa parviflora Desf. (1)

Stipa tenacissima L. $(1,2,3,4,5)$

\section{GNÉTACÉES}

Ephedra alata subsp. alenta Trab.(5)

GLOBULARIACÉES

Globularia alypum L. (1)

JUNCACÉES

Juncus sp. (5)

Juncus maritimus Lam. (5)

\section{LABIÉES}

Marrubium alyssoides Pomel (1)

Marrubium deserti Coss. $(3,4,5)$

Salvia aegyptica L.(4)

Salvia verbenaca subsp. clandestina Pugsl. $(2,3,4)$

Teucrium polium L. $(1,2)$

Thymus ciliatus Benth. (1)

Thymus sp. (5)

\section{LÉGUMINEUSES}

Adenocarpus bacquei Batt. \& Pitard.(5)

Astragalus armatus Willd. (1)
Astragalus epiglottis L. (3)

Astragalus gombiformis Pomel (5)

Genista saharae Coss. \& Dur. (5)

Medicago laciniata Mill. $(1,2,4)$

Ononis angustissima Batt. \& Trab. $(3,4,5)$

Retama retam Webb. $(4,5)$

Retama sphaerocarpa Boiss. (4)

LILIACÉES

Asphodelus microcarpus Viv. (1)

Asphodelus refractus Boiss. (5)

Asphodelus tenuifolius Cav. $(2,3)$

\section{MALVACÉES}

Malva aegyptica L. (4)

OLEACÉES

Olea europea L. (1)

OMBELLIFERES

Elaeoselinum thapsiodes Maire (1)

Eryngium ilicifolium Lam. $(3,5)$

Eryngium tricuspidatum L. $(1,2)$

Ferula cossoniana Bott. \& Trab. $(3,4,5)$

Pituranthos chloranthus Benth. \& Hook. $(4,5)$

Thapsia garganica L. (1)

\section{PALMACÉES}

Foenix dactylifera L. (5)

\section{PLANTAGINACÉES}

Plantago albicans L. $(2,3)$

Plantago amplexicaulis Cav. $(1,3,5)$

Plantago ovata Forsk. $(1,2)$

PLUMBAGINACÉES

Limoniastrum feei Batt. (5)

Limonium sinuatum subsp. beaumieranum Sauvage \& Vindt (5)

POLYGONACÉES

Rumex vesicarius L. (5)

RANUNCULACÉES

Adonis dentata Del.(3)

Ranunculus falcatus L. (2)

RESÉDACÉES

Randonia africana Coss. (5) 
Reseda lutea subsp lutea Maire $(1,2)$

RHAMNACÉES

Ziziphus lotus Lamk. (1, 2, 3, 4, 5)

SCROPHULARIACÉES

Antirrhinum ramosissimum Coss. \& Dur. (5)

Linaria aegyptica Dum (5)

TAMARICACÉES

Tamarix aphylla Korst. (5)

\section{TYMELEACÉES}

Thymelea microphylla Coss. \& Dur. $(3,4,5)$

Thymelea tartonraira All. $(1,2)$

\section{ZYGOPHYLLACÉES}

Fagonia cretica L. (2)

Fagonia glutinosa Delile $(2,5)$

Peganum harmala L. (1, 2, 3, 4, 5)

Zygophyllum sp. (5).

\section{BIBLIOGRAPHIE}

BOUKHRIS, M. -1973-Recherches écologiques et physiologiques sur les plantes gypsicoles de Tunisie. Thèse Doc. d'Etat, Univ. Sci. tech. Languedoc, Montpellier. 215p.

BOURAADA, K. - 1996- Le peuplement des végétaux et coléoptères des dunes fixées par des graminées vivaces dans le Maroc oriental. Thèse de 3ième cycle, Univ. Mohamed 1er, Fac. Sc. Oujda. 127p.

BRAUN BLANQUET, J. -1952- Prodrome des groupements végétaux de la France Méditerranée C.N.R.S. Ser. Carte group. veg. et dir. Carte group. veg. d'Afrique du Nord.

DJEBAILI, S. -1984- Recherches phytosociologiques et écologiques sur la végétation des hautes plaines steppiques et de l'Atlas Sahariens Algériens. Office des Publications Universitaires, Alger. 177p.

FOUCART, T. -1985-Analyse factorielle. Programmation sur micro-ordinateur. Ed. Masson.

GUINET, Ph. -1954- Carte de la Végétation de l'Algérie. Feuille de Beni Abbès au 1/200000. HALOUI, B. -1991- La végétation du Maroc oriental. Phytoécologie, Phytomasse Minéralomasse et Productivité des principaux écosystèmes forestiers. Thèse de Doc. es Sciences, Univ. Mohamed 1er, Fac. Sc. Oujda. 180p.

KAABECHE, M. -1996- La végétation steppique du Maghreb (Algérie, Maroc, Tunisie). Essai de synthèse phytosociologique par application des techniques numériques d'analyses. Documents phytosociologiques, Vol. XVI, Camerino.

KAHOUADJI, M.S. -1995-Contribution à une étude ehnobotanique dans le Maroc oriental. Thèse de 3ème cycle, Univ. Mohamed ler, Fac. Sc. Oujda.

LE HOUEROU, H.N. -1969- La végétation de la Tunisie steppique. Ann. Inst. Nat. Rech. Agrron. Tunis. $561 p$.

MAIRE, R. -1952/1977-Flore de l'Afrique du Nord, XIV vol. Edit. Lechevallier, Paris.

NEGRE, R. - 1959- Recherches phytogéographiques sur l'étage de végétation méditerranéen aride (sous étage chaud) au Maroc occidental. Trav. Inst. Sc. Cherif., Ser. Bot., 13, 385p.

NEGRE, R. -1961-Petite Flore des régions arides du Maroc occidental. C.N.R.S. Paris. 413p.

OZENDA, P. -1977-Flore du Sahara. C.N.R.S. Paris. 622p.

QUEZEL, P. -1999- Les grandes structures de végétation en région méditerranéene: Facteurs déterminants dans leur mise en place postglaciaire. Geobios, 32, $1:$ 19-32.

QUEZEL, P. et S. SANTA -1962/1963- Nouvelle Flore de l'Algérie et des régions désertiques méridionales. Tomes I et II. C.N.R.S. Paris.

THIOULOUSE, J. -1989- Statistical analysis and graphical display of multivariate data on the Macintosh. Computer applications in the bioscienses, 5, 4:287-292.

Aceptado para su publicación en junio de 2001

Direction des auteurs. Laboratoire d'Ecologie Végétale \& d'Aridoculture, UFR Sciences de l'environnement en milieu aride et semi-aride, Faculté des Sciences, Université Mohamed Premier, Oujda, Maroc. E-mail: maamri@sciences.univoujda.ac.ma 